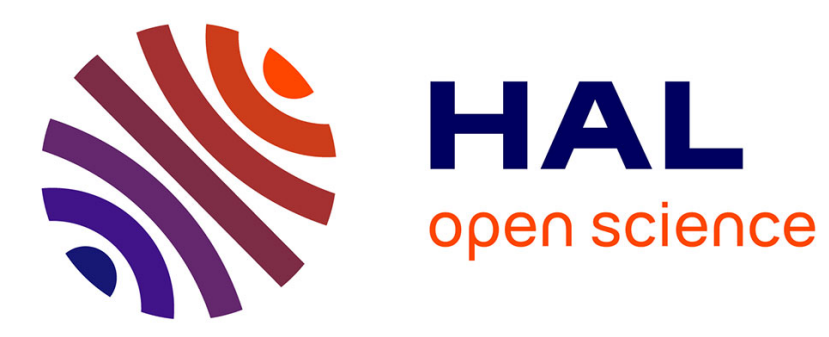

\title{
Avoiding approximate squares
}

Dalia Krieger, Pascal Ochem, Narad Rampersad, Jeffrey Shallit

\section{- To cite this version:}

Dalia Krieger, Pascal Ochem, Narad Rampersad, Jeffrey Shallit. Avoiding approximate squares. DLT 2007, 2007, Finland. pp.278-289. hal-00307153

\section{HAL Id: hal-00307153 https://hal.science/hal-00307153}

Submitted on 4 Nov 2008

HAL is a multi-disciplinary open access archive for the deposit and dissemination of scientific research documents, whether they are published or not. The documents may come from teaching and research institutions in France or abroad, or from public or private research centers.
L'archive ouverte pluridisciplinaire HAL, est destinée au dépôt et à la diffusion de documents scientifiques de niveau recherche, publiés ou non, émanant des établissements d'enseignement et de recherche français ou étrangers, des laboratoires publics ou privés. 


\title{
Avoiding Approximate Squares
}

\author{
Dalia Krieger ${ }^{2}$, Pascal Ochem ${ }^{1}$, Narad Rampersad $^{2}$, and Jeffrey Shallit ${ }^{2}$ \\ 1 LaBRI - Université Bordeaux 1 \\ 351, cours de la Libération \\ 33405 Talence Cedex FRANCE \\ ochem@labri.fr \\ 2 David R. Cheriton School of Computer Science \\ University of Waterloo \\ Waterloo, ON, N2L 3G1 CANADA \\ \{d2kriege,nrampersad\}@cs.uwaterloo.ca \\ shallit@graceland.uwaterloo.ca
}

\begin{abstract}
As is well-known, Axel Thue constructed an infinite word over a 3-letter alphabet that contains no squares, that is, no nonempty subwords of the form $x x$. In this paper we consider a variation on this problem, where we try to avoid approximate squares, that is, subwords of the form $x x^{\prime}$ where $|x|=\left|x^{\prime}\right|$ and $x$ and $x^{\prime}$ are "nearly" identical.
\end{abstract}

\section{Introduction}

A hundred years ago, Norwegian mathematician Axel Thue initiated the study of combinatorics on words $[13,14,2]$. One of his achievements was the construction of an infinite word over a three-letter alphabet that contains no squares, that is, no nonempty subwords of the form $x x$.

Many variations on this problem have been considered. For example, Brandenburg [3] and Dejean [5] considered the problem of avoiding fractional powers. A word $w$ is a $\alpha$-power if it can be written in the form $w=x^{n} y$, where $y$ is a prefix of $x$ and $\alpha=|w| /|x|$. A word $z$ contains an $\alpha$-power if some subword is a $\beta$-power, for $\beta \geq \alpha$; otherwise it avoids $\alpha$-powers. Similarly, a word $z$ contains an $\alpha^{+}$-power if some subword is a $\beta$-power for $\beta>\alpha$; otherwise it avoids $\alpha^{+}$-powers. We say $\alpha$-powers (resp. $\alpha^{+}$-powers) are avoidable over a $k$-letter alphabet if there exists an infinite word over that alphabet avoiding $\alpha$-powers (resp., $\alpha^{+}$-powers).

Dejean [5] improved Thue's result by showing how to avoid $(7 / 4)^{+}$-powers over a 3-letter alphabet; this result is optimal, as every ternary word of length $\geq 39$ contains a $7 / 4$-power. Pansiot [12] showed how to avoid $(7 / 5)^{+}$-powers over a 4-letter alphabet. Again, this is optimal, as every quaternary word of length $\geq 122$ contains a $7 / 5$-power.

Dejean also proved that for $k \geq 5$, one cannot avoid $k /(k-1)$-powers over a $k$-letter alphabet. She conjectured that it was possible to avoid $(k /(k-1))^{+}$powers over a $k$-letter alphabet. This conjecture was proved for $5 \leq k \leq 11$ by Moulin-Ollagnier, for $7 \leq k \leq 14$ by Mohammad-Noori and Currie [9], and for $k \geq 38$ by Carpi [4]. The cases $15 \leq k \leq 37$ remain open. 
Another variation is to avoid not all $\alpha$-powers, but only sufficiently long ones. Entringer, Jackson, and Schatz [6] showed how to construct a word over a 2-letter alphabet that avoids squares $x x$ with $|x| \geq 3$; here the number 3 is best possible.

In this paper we consider yet another variation, but one that seems natural: we consider avoiding approximate squares, that is, subwords of the form $x x^{\prime}$ where $x^{\prime}$ is "almost the same" as $x$. The precise definitions are given below. One of our main results is a further strengthening of Dejean's improvement on Thue for 3 letters.

Approximate squares (also known as approximate tandem repeats in the biological literature) have been studied before, but from the algorithmic point of view. Landau and Schmidt [7] and Kolpakov and Kucherov [8] both gave efficient algorithms for finding approximate squares in a string.

Notation: we use $\Sigma_{k}$ to denote the alphabet of $k$ letters $\{0,1,2, \ldots, k-1\}$.

\section{Approximate squares}

There are at least two natural notions of approximate square. We define them below.

For words $x, x^{\prime}$ of the same length, define the Hamming distance $d\left(x, x^{\prime}\right)$ as the number of positions in which $x$ and $x^{\prime}$ differ. For example, $d(01203,11002)=$ 2. We say that a word $x x^{\prime}$ with $|x|=\left|x^{\prime}\right|$ is a c-approximate square if $d\left(x, x^{\prime}\right) \leq c$. Using this terminology, for example, a 0 -approximate square is a square, and a 1-approximate square is either a square or differs from a square in exactly one position.

To avoid $c$-approximate squares, we would like to enforce the condition $d\left(x, x^{\prime}\right)>c$ for all $x, x^{\prime}$ of the same length, but clearly this is impossible if $|x| \leq c$. To avoid this technicality, we say a word $z$ avoids c-approximate squares if for all its subwords $x x^{\prime}$ where $|x|=\left|x^{\prime}\right|$ we have $d\left(x, x^{\prime}\right) \geq \min (c+1,|x|)$.

This definition is an "additive" version; there is also a "multiplicative" version. Given two words $x, x^{\prime}$ of the same length, we define their similarity $s\left(x, x^{\prime}\right)$ as the fraction of the number of positions in which $x$ and $x^{\prime}$ agree. Formally,

$$
s\left(x, x^{\prime}\right):=\frac{|x|-d\left(x, x^{\prime}\right)}{|x|} .
$$

Thus for example, $s(123456,101406)=1 / 2$. The similarity of a finite word $z$ is defined to be $\alpha=\max _{\substack{x, x^{\prime} \text { subwords of } z \\|x|=\left|x^{\prime}\right|}} s\left(x, x^{\prime}\right)$; we say such a word is $\alpha$-similar. Thus, a 1-similar finite word contains a square.

For infinite words, the situation is slightly more subtle. We say an infinite word $\mathbf{z}$ is $\alpha$-similar if $\alpha=\sup _{x, x^{\prime} \text { subwords of } \mathbf{z}} s\left(x, x^{\prime}\right)$ and there exists at least one subword $x x^{\prime}$ with $|x|=\left|x^{\prime}\right|$ and $\mid x\left(x, x^{\prime}\right)=\alpha$. Otherwise, if $\alpha=$ $\sup _{\substack{x, x^{\prime} \text { subwords of } \mathbf{z} \\|x|=\left|x^{\prime}\right|}} s\left(x, x^{\prime}\right)$, but $\alpha$ is not attained by any subword $x x^{\prime}$ of $\mathbf{z}$, then we say $\mathbf{z}$ is $\alpha^{-}$-similar. 
As an example, consider the infinite word over $\Sigma_{3}$,

$$
\mathbf{c}=210201210120210201202 \cdots
$$

defined to be the length of contiguous blocks of 1's between consecutive 0's in the Thue-Morse sequence $\mathbf{t}$. As is well-known, $\mathbf{c}$ is square-free, so it cannot be 1-similar. However, since $\mathbf{t}$ contains arbitrarily large squares, it follows that $\mathbf{c}$ must contain arbitrarily large 1 -approximate squares, and so $\mathbf{c}$ is $1^{-}$-similar.

\section{Words of low similarity}

The main problem of interest is,

Given an alphabet $\Sigma$ of size $k$, what is the smallest similarity possible over all infinite words over $\Sigma$ ? We call this the similarity coefficient of $k$.

Answering this question has two aspects. We can explicitly construct an infinite word that is $\alpha$-similar (or $\alpha^{-}$-similar). To show that $\alpha$ is best possible, we can construct a tree of all finite words that are $\beta$-similar for $\beta<\alpha$. The root of this tree is labeled 0 (which suffices by symmetry), and if a node is labeled $w$, its children are labeled $w a$ for all $a \in \Sigma$. If a node is $\beta$-similar for some $\beta \geq \alpha$, it becomes a leaf and no children are added. We can then use depth-first or breadth-first search to explore this tree. The number of leaves of this tree represents the (finite) number of words beginning with 0 that are $\beta$-similar for $\beta<\alpha$, and the height $h$ of the tree is the length of the longest words with this property. The number of leaves at depth $h$ represent the number of maximal words beginning with 0 that are $\beta$ similar for some $\beta<\alpha$.

We performed this computation for various alphabet sizes $k$, and the results are reported below in Table 1 . For $k=8$, our method took advantage of some symmetries to speed up the computation, and as a result, we did not compute the number of leaves or maximal strings. For the reported values, these computations represent a proof that the similarity coefficient is at least as large as the $\alpha$ reported.

\begin{tabular}{|c|c|c|c|c|c|}
\hline $\begin{array}{c}\text { Alphabet } \\
\text { Size } k\end{array}$ & $\begin{array}{c}\text { Similarity } \\
\text { Coefficient } \\
\alpha\end{array}$ & $\begin{array}{r}\text { Height } \\
\text { of } \\
\text { Tree }\end{array}$ & \begin{tabular}{|r|} 
Number \\
of \\
Leaves
\end{tabular} & $\begin{array}{r}\text { Number of } \\
\text { Maximal } \\
\text { Words }\end{array}$ & \begin{tabular}{l|l} 
& Lexicographically \\
First Maximal Words
\end{tabular} \\
\hline 2 & 1 & 3 & 4 & 1 & 010 \\
\hline 3 & $3 / 4$ & 41 & 2475 & 36 & 01020120210120102120121020120210120102101 \\
\hline 4 & $1 / 2$ & 9 & 382 & 6 & 012310213 \\
\hline 5 & $2 / 5$ & 75 & 3902869 & 48 & \begin{tabular}{|l|l}
12304310342041340120314210412342012403410230420312340321024320410342140243
\end{tabular} \\
\hline 6 & $1 / 3$ & 17 & 342356 & 480 & 01234150325143012 \\
\hline 7 & $?$ & $?$ & & $?$ & $?$ \\
\hline 8 & $1 / 4$ & 71 & - & - & 01234056731460251647301275634076213574102364075120435674103271564073142 \\
\hline
\end{tabular}

Table 1. Similarity bounds 
For alphabet size $k=2$, every infinite word is 1 -similar. We now report on larger alphabet sizes.

Theorem 1. There exists an infinite 3/4-similar word $\mathbf{w}$ over $\{0,1,2\}$.

Proof. Let $h$ be the 24-uniform morphism defined by

$$
\begin{aligned}
& 0 \rightarrow 012021201021012102120210 \\
& 1 \rightarrow 120102012102120210201021 \\
& 2 \rightarrow 201210120210201021012102 .
\end{aligned}
$$

The following lemma may be verified computationally.

Lemma 1. Let $a, b, c \in\{0,1,2\}, a \neq b$. Let $w$ be any subword of length 24 of $h(a b)$. If $w$ is neither a prefix nor a suffix of $h(a b)$, then $h(c)$ and $w$ mismatch in at least 9 positions.

Let $\mathbf{w}=h^{\omega}(0)$. We shall show that $\mathbf{w}$ has the desired property. We argue by contradiction. Suppose that $\mathbf{w}$ contains a subword $y y^{\prime}$ with $|y|=\left|y^{\prime}\right|$ such that $y$ and $y^{\prime}$ match in more than $3 / 4 \cdot|y|$ positions. Let us suppose further that $|y|$ is minimal.

We may verify computationally that $\mathbf{w}$ contains no such subword $y y^{\prime}$ where $|y| \leq 72$. We therefore assume from now on that $|y|>72$.

Let $w=a_{1} a_{2} \cdots a_{n}$ be a word of minimal length such that $h(w)=x y y^{\prime} z$ for some $x, z \in\{0,1,2\}^{*}$. By the minimality of $w$, we have $0 \leq|x|,|z|<24$.

For $i=1,2, \ldots, n$, define $A_{i}=h\left(a_{i}\right)$. Then if $h(w)=x y y^{\prime} z$, we can write

$$
h(w)=A_{1} A_{2} \cdots A_{n}=A_{1}^{\prime} A_{1}^{\prime \prime} A_{2} \cdots A_{j-1} A_{j}^{\prime} A_{j}^{\prime \prime} A_{j+1} \cdots A_{n-1} A_{n}^{\prime} A_{n}^{\prime \prime}
$$

where

$$
\begin{aligned}
A_{1} & =A_{1}^{\prime} A_{1}^{\prime \prime} \\
A_{j} & =A_{j}^{\prime} A_{j}^{\prime \prime} \\
A_{n} & =A_{n}^{\prime} A_{n}^{\prime \prime} \\
x & =A_{1}^{\prime} \\
y & =A_{1}^{\prime \prime} A_{2} \cdots A_{j-1} A_{j}^{\prime} \\
y^{\prime} & =A_{j}^{\prime \prime} A_{j+1} \cdots A_{n-1} A_{n}^{\prime} \\
z & =A_{n}^{\prime \prime},
\end{aligned}
$$

and $\left|A_{1}^{\prime \prime}\right|,\left|A_{j}^{\prime \prime}\right|>0$. See Figure 1 .

If $\left|A_{1}^{\prime \prime}\right|>\left|A_{j}^{\prime \prime}\right|$, then, writing $y$ and $y^{\prime}$ atop one another, as illustrated in Figure 2, one observes that for $t=j+1, j+2, \ldots, n-1$, each $A_{t}$ "lines up" with a subword, say $B_{t}$, of $A_{t-j} A_{t-j+1}$. We now apply Lemma 1 to conclude that each $A_{t}$ mismatches with $B_{t}$ in at least 9 of 24 positions. Consequently, $y$ and $y^{\prime}$ mismatch in at least $9(j-2)$ positions. Since $j \geq|y| / 24+1$, we have that 


\begin{tabular}{|c|c|c|c|c|c|c|c|c|}
\hline$A_{1}^{\prime} A_{1}^{\prime \prime}$ & & & & \begin{tabular}{l|l}
$A_{j}^{\prime}$ & $A_{j}^{\prime \prime}$ \\
\end{tabular} & & & & \begin{tabular}{|l|l|l}
$A_{n}^{\prime}$ & $A_{n}^{\prime \prime}$ \\
\end{tabular} \\
\hline$A_{1}$ & $A_{2}$ & $\ldots$ & $A_{j-1}$ & $A_{j}$ & $A_{j+1}$ & $\cdots$ & $A_{n-1}$ & $A_{n}$ \\
\hline$x$ & & & & & & $y^{\prime}$ & & $z$ \\
\hline
\end{tabular}

Fig. 1. The string $x y y^{\prime} z$ within $h(w)$

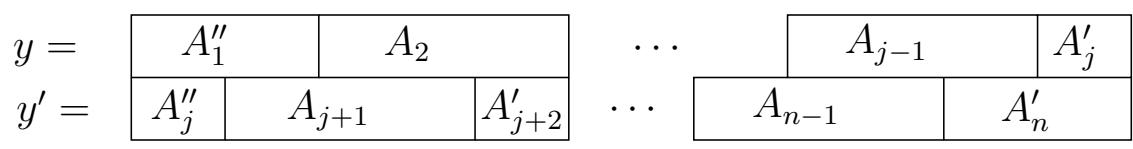

Fig. 2. The case $\left|A_{1}^{\prime \prime}\right|>\left|A_{j}^{\prime \prime}\right|$

$9(j-2) \geq 9(|y| / 24-1)$. However, $9(|y| / 24-1)>|y| / 4$ for $|y|>72$, so that $y$ and $y^{\prime}$ mismatch in more than $1 / 4 \cdot|y|$ positions, contrary to our assumption.

If $\left|A_{1}^{\prime \prime}\right|<\left|A_{j}^{\prime \prime}\right|$, as illustrated in Figure 3 , then a similar argument shows that $y$ and $y^{\prime}$ mismatch in more than $1 / 4 \cdot|y|$ positions, contrary to our assumption.

\begin{tabular}{|c|c|c|c|c|c|c|}
\hline$y=$ & $A_{1}^{\prime \prime}$ & $A_{2}$ & $A_{3}^{\prime}$ & \multirow{2}{*}{$\begin{array}{c}\cdots \\
\cdots\end{array}$} & $A_{j-1}$ & $A_{j}^{\prime}$ \\
\hline$y^{\prime}=$ & $A_{j}^{\prime \prime}$ & & & & \begin{tabular}{l|l} 
& $A_{n-1}$
\end{tabular} & $A_{n}^{\prime}$ \\
\hline
\end{tabular}

Fig. 3. The case $\left|A_{1}^{\prime \prime}\right|<\left|A_{j}^{\prime \prime}\right|$

Therefore $\left|A_{1}^{\prime \prime}\right|=\left|A_{j}^{\prime \prime}\right|$. We first observe that any pair of words taken from $\{h(0), h(1), h(2)\}$ mismatch at every position. We now consider several cases.

Case 1: $A_{1}=A_{j}=A_{n}$. Then letting $u=A_{1} A_{2} \cdots A_{j-1}$ and $u^{\prime}=A_{j} A_{j+1} \cdots A_{n-1}$, we see that $u$ and $u^{\prime}$ match in exactly the same number of positions as $y$ and $y^{\prime}$.

Case 2: $A_{1}=A_{j} \neq A_{n}$. Then letting $u=A_{1} A_{2} \cdots A_{j-1}$ and $u^{\prime}=A_{j} A_{j+1} \cdots A_{n-1}$, we see that $u$ and $u^{\prime}$ match in at least as many positions as $y$ and $y^{\prime}$.

Case 3: $A_{1} \neq A_{j}=A_{n}$. Then letting $u=A_{2} A_{3} \cdots A_{j}$ and $u^{\prime}=A_{j+1} A_{j+2} \cdots A_{n}$, we see that $u$ and $u^{\prime}$ match in at least as many positions as $y$ and $y^{\prime}$.

Case 4: $A_{1}=A_{n} \neq A_{j}$. Then letting $u=A_{1} A_{2} \cdots A_{j-1}$ and $u^{\prime}=A_{j} A_{j+1} \cdots A_{n-1}$, we see that $u$ and $u^{\prime}$ match in exactly the same number of positions as $y$ and $y^{\prime}$.

Case 5: $A_{1}, A_{j}$, and $A_{n}$ are all distinct. Then letting $u=A_{1} A_{2} \cdots A_{j-1}$ and $u^{\prime}=A_{j} A_{j+1} \cdots A_{n-1}$, we see that $u$ and $u^{\prime}$ match in exactly the same number of positions as $y$ and $y^{\prime}$.

We finish the argument by considering the word $u u^{\prime}$. First observe that either

$$
u u^{\prime}=h\left(a_{1} a_{2} \cdots a_{j-1}\right) h\left(a_{j} a_{j+1} \cdots a_{n-1}\right)
$$


or

$$
u u^{\prime}=h\left(a_{2} a_{3} \cdots a_{j}\right) h\left(a_{j+1} a_{j+2} \cdots a_{n}\right) .
$$

Without loss of generality, let us assume that the first case holds.

Recall our previous observation that the words $h(0), h(1)$, and $h(2)$ have distinct letters at every position. Suppose then that there is a mismatch between $u$ and $u^{\prime}$ occurring within blocks $A_{t}$ and $A_{t+j}$ for some $t, 1 \leq t \leq j$. Then $A_{t}$ and $A_{t+j}$ mismatch at every position. Moreover, we have $a_{j} \neq a_{j+t}$. Conversely, if $A_{t}$ and $A_{t+j}$ match at any single position, then they match at every position, and we have $a_{t}=a_{t+j}$.

Let $v=a_{1} a_{2} \cdots a_{j-1}$ and $v^{\prime}=a_{j} a_{j+1} \cdots a_{n-1}$. Let $m$ be the number of matches between $u$ and $u^{\prime}$. From our previous observations we deduce that the number of matches $m^{\prime}$ between $v$ and $v^{\prime}$ is $m / 24$, but since $|v|=|u| / 24, m^{\prime} /|v|=$ $m /|u|$. Thus, if $m /|u|>3 / 4$, as we have assumed, then $m^{\prime} /|v|>3 / 4$. But the set $\{h(0), h(1), h(2)\}$ is a code, so that $v v^{\prime}$ is the unique pre-image of $u u^{\prime}$. The word $v v^{\prime}$ is thus a subword of $\mathbf{w}$, contradicting the assumed minimality of $y y^{\prime}$. We conclude that no such $y y^{\prime}$ occurs in $\mathbf{w}$, and this completes the argument that $\mathbf{w}$ is $3 / 4$-similar.

Next, we consider the case $k=4$.

Theorem 2. There exists an infinite $1 / 2$-similar word $\mathbf{x}$ over $\{0,1,2,3\}$.

Proof. Let $g$ be the 36-uniform morphism defined by

$$
\begin{aligned}
& 0 \rightarrow 012132303202321020123021203020121310 \\
& 1 \rightarrow 123203010313032131230132310131232021 \\
& 2 \rightarrow 230310121020103202301203021202303132 \\
& 3 \rightarrow 301021232131210313012310132313010203 .
\end{aligned}
$$

Then $\mathbf{x}=g^{\omega}(0)$ has the desired property. The proof is entirely analogous to that of Theorem 1 and is omitted.

In our last result of this section, we show that we can obtain infinite words of arbitrarily low similarity, provided the alphabet size is sufficiently large. The main tool is the following [1, Lemma 5.1.1]:

Lemma 2 (Lovász Local Lemma; asymmetric version). Let I be a finite set, and let $\left\{A_{i}\right\}_{i \in I}$ be events in a probability space. Let $E$ be a set of pairs $(i, j) \in I \times I$ such that $A_{i}$ is mutually independent of all the events $\left\{A_{j}:(i, j) \notin\right.$ $E\}$. Suppose there exist real numbers $\left\{x_{i}\right\}_{i \in I}, 0 \leq x_{i}<1$, such that for all $i \in I$,

$$
\operatorname{Prob}\left(A_{i}\right) \leq x_{i} \prod_{(i, j) \in E}\left(1-x_{j}\right) .
$$

Then

$$
\operatorname{Prob}\left(\bigcap_{i \in I} \overline{A_{i}}\right) \geq \prod_{i \in I}\left(1-x_{i}\right)>0
$$


We now state our result.

Theorem 3. Let $c>1$ be an integer. There exists an infinite $1 / c$-similar word.

Proof. Let $k$ and $N$ be positive integers, and let $w=w_{1} w_{2} \cdots w_{N}$ be a random word of length $N$ over a $k$-letter alphabet $\Sigma$. Here each letter of $w$ is chosen uniformly and independently at random from $\Sigma$.

Let

$$
I=\{(t, r): 0 \leq t<N, 1 \leq r \leq\lfloor(N-t) / 2\rfloor\} .
$$

For $i=(t, r) \in I$, write $y=w_{t} \cdots w_{t+r-1}$ and $y^{\prime}=w_{t+r} \cdots w_{t+2 r-1}$. Let $A_{i}$ denote the event $s\left(y, y^{\prime}\right)>1 / c$. A crude overestimate of $\operatorname{Prob}\left(A_{i}\right)$ is

$$
\begin{aligned}
\operatorname{Prob}\left(A_{i}\right) & \leq \frac{\left(\begin{array}{c}
r \\
\lceil r / c\rceil+1
\end{array}\right) k^{\lceil r / c\rceil+1} k^{2 r-2(\lceil r / c\rceil+1)}}{k^{2 r}} \\
& \leq\left(\begin{array}{c}
r \\
\lceil r / 2\rceil
\end{array}\right) k^{-r / c} \leq 2^{r} k^{-r / c},
\end{aligned}
$$

where the last inequality comes from Stirling's approximation.

For all positive integers $r$, define $\xi_{r}=2^{-2 r}$. For any real number $\alpha \leq 1 / 2$, we have $(1-\alpha) \geq e^{-2 \alpha}$. Hence, $\left(1-\xi_{r}\right) \geq e^{-2 \xi_{r}}$. For $i=(t, r) \in I$, define $x_{i}=\xi_{r}$. Let $E$ be as in the local lemma. Note that a subword of length $2 r$ of $w$ overlaps with at most $2 r+2 s-1$ subwords of length $2 s$. Thus, for all $i=(t, r) \in I$, we have

$$
\begin{aligned}
x_{i} \prod_{(i, j) \in E}\left(1-x_{j}\right) & \geq \xi_{r} \prod_{s=1}^{\lfloor N / 2\rfloor}\left(1-\xi_{s}\right)^{2 r+2 s-1} \geq \xi_{r} \prod_{s=1}^{\infty}\left(1-\xi_{s}\right)^{2 r+2 s-1} \\
& \geq \xi_{r} \prod_{s=1}^{\infty} e^{-2 \xi_{s}(2 r+2 s-1)} \geq 2^{-2 r} \prod_{s=1}^{\infty} e^{-2\left(2^{-2 s}\right)(2 r+2 s-1)} \\
& \geq 2^{-2 r} \exp \left[-2\left(2 r \sum_{s=1}^{\infty} \frac{1}{2^{2 s}}+\sum_{s=1}^{\infty} \frac{2 s-1}{2^{2 s}}\right)\right] \\
& \geq 2^{-2 r} \exp \left[-2\left(2 r\left(\frac{1}{3}\right)+\frac{5}{9}\right)\right] \\
& \geq 2^{-2 r} \exp \left(-\frac{4}{3} r-\frac{10}{9}\right) .
\end{aligned}
$$

The hypotheses of the local lemma are met if $2^{r} k^{-r / c} \leq 2^{-2 r} \exp \left(-\frac{4}{3} r-\frac{10}{9}\right)$. Taking logarithms, we require $r \log 2-\frac{r}{c} \log k \leq-2 r \log 2-\frac{4}{3} r-\frac{10}{9}$. Rearranging terms, we require $c\left(3 \log 2+\frac{4}{3}+\frac{10}{9 r}\right) \leq \log k$. The left side of this inequality is largest when $r=1$, so we define $d_{1}=3 \log 2+\frac{4}{3}+\frac{10}{9}$, and insist that $c \cdot d_{1} \leq \log k$. Hence, for $k \geq e^{c \cdot d_{1}}$, we may apply the local lemma to conclude that with positive probability, $w$ is $1 / c$-similar. Since $N=|w|$ is arbitrary, we conclude that there are arbitrarily large such $w$. By König's Infinity Lemma, there exists an infinite $1 / c$-similar word, as required. 


\section{Words avoiding $c$-approximate squares}

In this section we consider the "additive" version of the problem. Table 2 reflects our results using a backtracking algorithm: there is no infinite word over a $k$ letter alphabet that avoids $c$-approximate squares, for the $k$ and $c$ given below.

\begin{tabular}{|c|c|c|c|c|c|}
\hline $\begin{array}{l}\text { Alphabet } \\
\text { Size } k\end{array}$ & $c$ & $\begin{array}{r}\text { Height } \\
\text { of } \\
\text { Tree }\end{array}$ & $\begin{array}{r}\text { Number } \\
\text { of } \\
\text { Leaves }\end{array}$ & $\begin{array}{r}\text { Number of } \\
\text { Maximal } \\
\text { Words }\end{array}$ & $\begin{array}{l}\text { Lexicographically } \\
\text { First Maximal Words }\end{array}$ \\
\hline 2 & 0 & 4 & 3 & 1 & 010 \\
\hline 3 & 1 & 5 & 23 & 2 & 01201 \\
\hline 4 & 2 & 7 & 184 & 6 & 0123012 \\
\hline 5 & 2 & 11 & 3253 & 24 & 01234102314 \\
\hline 6 & 3 & 11 & 35756 & 960 & 01234051230 \\
\hline 7 & 4 & 13 & 573019 & 6480 & 0123450612340 \\
\hline 8 & 5 & 15 & - & - & 012345607123450 \\
\hline
\end{tabular}

Theorem 4. There is an infinite word over a 3-letter alphabet that avoids 0approximate squares, and the 0 is best possible.

Proof. Any ternary word avoiding squares, such as the fixed point, starting with 2 , of $2 \rightarrow 210,1 \rightarrow 20,0 \rightarrow 1$, satisfies the conditions of the theorem. The result is best possible, from Table 2 .

Theorem 5. There is an infinite word over a 4-letter alphabet that avoids 1approximate squares, and the 1 is best possible.

Proof. Let $\mathbf{c}$ be any squarefree word over $\{0,1,2\}$, and consider the image under the 48-uniform morphism $\gamma$ defined by

$$
\begin{aligned}
& 0 \rightarrow 012031023120321031201321032013021320123013203123 \\
& 1 \rightarrow 012031023120321023103213021032013210312013203123 \\
& 2 \rightarrow 012031023012310213023103210231203210312013203123
\end{aligned}
$$

The resulting word $\mathbf{d}=\gamma(\mathbf{c})$ avoids 1-approximate squares. The result is best possible, from Table 2 .

The proof is similar to that of Theorem 1. Suppose to the contrary that $\mathbf{d}$ contains a 1-approximate square $y y^{\prime},|y|=\left|y^{\prime}\right|$. We may verify computationally that $\mathbf{d}$ contains no such subword $y y^{\prime}$ where $|y| \leq 96$. We therefore assume from now on that $|y|>96$.

Let $w=a_{1} a_{2} \cdots a_{n}$ be a word of minimal length such that $\gamma(w)=x y y^{\prime} z$ for some $x, z \in\{0,1,2,3\}^{*}$. By the minimality of $w$, we have $0 \leq|x|,|z|<48$. 
For $i=1,2, \ldots, n$, define $A_{i}=\gamma\left(a_{i}\right)$. Just as in the proof of Theorem 1, we write

$$
\gamma(w)=A_{1} A_{2} \cdots A_{n}=A_{1}^{\prime} A_{1}^{\prime \prime} A_{2} \cdots A_{j-1} A_{j}^{\prime} A_{j}^{\prime \prime} A_{j+1} \cdots A_{n-1} A_{n}^{\prime} A_{n}^{\prime \prime},
$$

so that the situation illustrated in Figure 1 applies to $x y y^{\prime} z$ within $\gamma(w)$. We now make the following observations regarding the morphism $\gamma$ :

1. Let $a, b, c \in\{0,1,2\}, a \neq b$. Let $u$ be any subword of length 48 of $\gamma(a b)$. If $u$ is neither a prefix nor a suffix of $\gamma(a b)$, then $\gamma(c)$ and $u$ mismatch in at least 18 positions.

2. Let $a, b \in\{0,1,2\}, a \neq b$. Then $\gamma(a)$ and $\gamma(b)$ mismatch in at least 18 positions.

3. Let $u, u^{\prime}, v, v^{\prime}$ be words satisfying the following:

$-|u|=\left|u^{\prime}\right|,|v|=\left|v^{\prime}\right|$, and $|u v|=\left|u^{\prime} v^{\prime}\right|=48$

- each of $u$ and $u^{\prime}$ is a suffix of a word in $\{\gamma(0), \gamma(1), \gamma(2)\}$; and

- each of $v$ and $v^{\prime}$ is a prefix of a word in $\{\gamma(0), \gamma(1), \gamma(2)\}$.

Then either $u v=u^{\prime} v^{\prime}$ or $u v$ and $u^{\prime} v^{\prime}$ mismatch in at least 18 positions.

4. Let $a \in\{0,1,2\}$. Then $\gamma(a)$ is uniquely determined by either its prefix of length 17 or its suffix of length 17 .

From the first observation, we deduce, as in the proof of Theorem 1, that the cases illustrated by Figures 2 and 3 cannot occur. In particular, we have that $\left|A_{1}^{\prime \prime}\right|=\left|A_{j}^{\prime \prime}\right|$ and $\left|A_{j}^{\prime}\right|=\left|A_{n}^{\prime}\right|$.

From the second observation, we deduce that for $i=2,3, \ldots, j-1, A_{i}=$ $A_{i+j-1}$, and consequently, $a_{i}=a_{i+j-1}$.

From the third observation, we deduce that $A_{1}^{\prime \prime}=A_{j}^{\prime \prime}$ and $A_{j}^{\prime}=A_{n}^{\prime}$.

From the fourth observation, we deduce that either $A_{1}=A_{j}$ or $A_{j}=A_{n}$. If $A_{1}=A_{j}$, then $a_{1}=a_{j}$; if $A_{j}=A_{n}$, then $a_{j}=a_{n}$. In the first case, $a_{1} a_{2} \cdots a_{j-1} a_{j} a_{j+1} \cdots a_{n-1}$ is a square in c, contrary to our assumption. In the second case, $a_{2} a_{3} \cdots a_{j} a_{j+1} a_{j+2} \cdots a_{n}$ is a square in $\mathbf{c}$, contrary to our assumption.

We conclude that $\mathbf{d}$ contains no 1-approximate square $y y^{\prime}$, as required.

Theorem 6. There is an infinite word over a 6-letter alphabet that avoids 2approximate squares, and the 2 is best possible.

Proof. Let $\mathbf{c}$ be any squarefree word over $\{0,1,2\}$, and consider the image under the 6 -uniform morphism $\beta$ defined by

$$
0 \rightarrow 012345 ; \quad 1 \rightarrow 012453 ; \quad 2 \rightarrow 012534 .
$$

The resulting word avoids 2-approximate squares. The result is best possible, from Table 2 .

The proof is similar to that of Theorem 5 , so we only note the properties of the morphism $\beta$ needed to derive the result:

1. Let $a, b, c \in\{0,1,2\}, a \neq b$. Let $u$ be any subword of length 6 of $\beta(a b)$. If $u$ is neither a prefix nor a suffix of $\beta(a b)$, then $\beta(c)$ and $u$ mismatch in at least 3 positions. 
2. Let $a, b \in\{0,1,2\}, a \neq b$. Then $\beta(a)$ and $\beta(b)$ mismatch in at least 3 positions.

3. Let $u, u^{\prime}, v, v^{\prime}$ be words satisfying the following:

- $|u|=\left|u^{\prime}\right|,|v|=\left|v^{\prime}\right|$, and $|u v|=\left|u^{\prime} v^{\prime}\right|=6$;

- each of $u$ and $u^{\prime}$ is a suffix of a word in $\{\beta(0), \beta(1), \beta(2)\}$; and

- each of $v$ and $v^{\prime}$ is a prefix of a word in $\{\beta(0), \beta(1), \beta(2)\}$.

Then either $u v=u^{\prime} v^{\prime}$ or $u v$ and $u^{\prime} v^{\prime}$ mismatch in at least 3 positions.

4. Let $a \in\{0,1,2\}$. Then $\beta(a)$ is uniquely determined by either its prefix of length 4 or its suffix of length 1.

Further results on additive similarity are summarized in the next theorem.

Theorem 7. For each $k, n, d$ given below, there is an infinite word over a $k$ letter alphabet that avoids n-approximate squares, and in each case such an infinite word can be generated by applying the given d-uniform morphism to any infinite squarefree word over $\{0,1,2\}$. (Note that we have used the coding $A=10$, $B=11$, etc. )

\begin{tabular}{|c|c|c|c|}
\hline$k$ & $n$ & & Morphism \\
\hline \multirow[t]{3}{*}{7} & 3 & 14 & $0 \rightarrow 01234056132465$ \\
\hline & & & $1 \rightarrow 01234065214356$ \\
\hline & & & $2 \rightarrow 01234510624356$ \\
\hline \multirow[t]{3}{*}{8} & 4 & 16 & $0 \rightarrow 0123456071326547$ \\
\hline & & & $1 \rightarrow 0123456072154367$ \\
\hline & & & $2 \rightarrow 0123456710324765$ \\
\hline \multirow[t]{3}{*}{9} & 5 & 36 & $0 \rightarrow 012345607821345062718345670281346578$ \\
\hline & & & $1 \rightarrow 012345607182346750812347685102346578$ \\
\hline & & & $2 \rightarrow 012345607182346510872345681702346578$ \\
\hline \multirow[t]{3}{*}{11} & 6 & 20 & $0 \rightarrow 012345670 \mathrm{~A} 812954768 \mathrm{~A}$ \\
\hline & & & $1 \rightarrow 0123456709 \mathrm{~A} 1843576 \mathrm{~A} 9$ \\
\hline & & & $2 \rightarrow 01234567089 A 24365798$ \\
\hline \multirow[t]{3}{*}{12} & 7 & 24 & $0 \rightarrow 012345678091 \mathrm{AB} 2354687 \mathrm{~A} 9 \mathrm{~B}$ \\
\hline & & & $1 \rightarrow 012345678091 \mathrm{~A} 3 \mathrm{~B} 4257689 \mathrm{AB}$ \\
\hline & & & $2 \rightarrow 012345678091 \mathrm{~A} 2 \mathrm{~B} 3465798 \mathrm{AB}$ \\
\hline \multirow[t]{3}{*}{13} & 8 & 26 & $0 \rightarrow 01234567890 \mathrm{~A} 1 \mathrm{BC} 24635798 \mathrm{BAC}$ \\
\hline & & & $1 \rightarrow 01234567890 \mathrm{~A} 1 \mathrm{~B} 3 \mathrm{C} 4257689 \mathrm{ABC}$ \\
\hline & & & $2 \rightarrow 01234567890 \mathrm{~A} 1 \mathrm{~B} 2 \mathrm{C} 354687 \mathrm{~A} 9 \mathrm{BC}$ \\
\hline \multirow[t]{3}{*}{14} & 9 & 28 & $0 \rightarrow$ 0123456789A0B1DC32465798BDAC \\
\hline & & & $1 \rightarrow$ 0123456789A0B1DC243576A98DBC \\
\hline & & & $2 \rightarrow 0123456789 \mathrm{AOB} 1 \mathrm{CD} 325468 \mathrm{~A} 79 \mathrm{CBD}$ \\
\hline \multirow[t]{3}{*}{15} & 10 & 30 & $0 \rightarrow$ 0123456789AB0D1CE3246579B8ACDE \\
\hline & & & $1 \rightarrow$ 0123456789AB0D1CE2435768A9DCBE \\
\hline & & & $2 \rightarrow$ 0123456789AB0CED32154687BA9DEC \\
\hline
\end{tabular}


In each case, the proof is similar to the ones given previously, and is omitted.

Theorem 8. For all integers $n \geq 3$, there is an infinite word over an alphabet of $2 n$ letters that avoids $(n-1)$-approximate squares.

Proof. Consider the $2 n$-uniform morphism $h: \Sigma_{3}^{*} \rightarrow \Sigma_{2 n}^{*}$ defined as follows:

$$
\begin{aligned}
& 0 \rightarrow 012 \cdots(n-1) n \cdots(2 n-1) \\
& 1 \rightarrow 012 \cdots(n-1)(n+1)(n+2) \cdots(2 n-1) n \\
& 2 \rightarrow 012 \cdots(n-1)(n+2)(n+3) \cdots(2 n-1) n(n+1)
\end{aligned}
$$

We claim that if $\mathbf{w}$ is any squarefree word over $\Sigma_{3}$, then $h(\mathbf{w})$ has the desired properties. The proof is a simple generalization of Theorem 6 .

\section{Another variation}

Yet another variation we can study is trying to avoid $x x^{\prime}$ where $x$ is very similar to $x^{\prime}$, but only for sufficiently large $x$. Let us say that a finite word is $(n, \alpha)$ similar if $\alpha=\sup _{\substack{x, x^{\prime} \text { subwords of } \\|x|=\left|x^{\prime}\right| \geq n}} s\left(x, x^{\prime}\right)$, and analogous definitions for infinite $z$.

Exercise 5.8.1 of Alon and Spencer [1] asks the reader to show, using the Lovász local lemma that, (in our language) for every $\epsilon>0$, there exists an infinite binary word $\mathbf{z}$ and an integer $c$ such that $\mathbf{z}$ is $(c, \alpha)$-similar for some $\alpha \leq \frac{1}{2}+\epsilon$.

Theorem 9. $\frac{1}{2}$ is best possible in the previous result.

Proof. Suppose $\frac{1}{2}$ is not best possible. Then there exists an infinite binary word $\mathbf{z}$ and a positive integer $c$, such that any subword $x x^{\prime}$ of $\mathbf{z}$ with $|x|=\left|x^{\prime}\right| \geq c$ satisfies $s\left(x, x^{\prime}\right)<\frac{1}{2}$. Consider a subword of $\mathbf{z}$ of the form $x x^{\prime} y y^{\prime}$, with $|x|=$ $\left|x^{\prime}\right|=|y|=\left|y^{\prime}\right|=c$. By our assumption, $s\left(x, x^{\prime}\right)<\frac{1}{2}$ and $s\left(x^{\prime}, y\right)<\frac{1}{2}$; hence, since $\mathbf{z}$ is defined over a binary alphabet, necessarily $s(x, y)>\frac{1}{2}$. Similarly, we must have $s\left(x^{\prime}, y^{\prime}\right)>\frac{1}{2}$. But then by definition of $s$,

$$
2 c \cdot s\left(x x^{\prime}, y y^{\prime}\right)=c \cdot s(x, y)+c \cdot s\left(x^{\prime}, y^{\prime}\right)>\frac{c}{2}+\frac{c}{2}=c,
$$

and so $s\left(x x^{\prime}, y y^{\prime}\right)>\frac{1}{2}$, a contradiction to our assumption.

\section{Edit distance}

There are many definitions of edit distance, but for our purposes, we say the edit distance $e(x, y)=c$ if $x$ can be transformed into $y$ by a sequence of $c$ insertions, deletions, or replacements, and no sequence of $c-1$ insertions, deletions, or replacements suffices.

We can expand our notion of approximate square to avoid all words that are within edit distance $c$ of all squares. 
For example, consider the case $c=1$. Then every word of length 1 , say $a$, is within edit distance 1 of a square, as we can simply insert $a$ to get $a a$. Similarly, every word of length 2 , say $a b$, is within edit distance 1 of a square, as we can simply replace the $b$ by $a$ to get $a a$. Thus we need to restrict our attention to avoiding words that are within edit distance $c$ of all sufficiently large squares.

Theorem 10. There is an infinite word over 5 letters such that all subwords $x$ with $|x| \geq 3$ are neither squares, nor within edit distance 1 of any square. There is no such word over 4 letters.

Proof. The usual tree traversal technique shows there is no such word over 4 letters Over 5 letters we can use the 5 -uniform morphism $h$ defined by

$$
0 \rightarrow 01234 ; \quad 1 \rightarrow 02142 ; \quad 2 \rightarrow 03143 .
$$

We claim the image of every square-free word under $h$ has the desired property. Details will appear in the final paper.

\section{References}

1. N. Alon and J. Spencer. The Probabilistic Method. 2nd edition. Wiley, 2000.

2. J. Berstel. Axel Thue's Papers on Repetitions in Words: a Translation. Number 20 in Publications du Laboratoire de Combinatoire et d'Informatique Mathématique. Université du Québec à Montréal, February 1995.

3. F.-J. Brandenburg. Uniformly growing $k$-th power-free homomorphisms. Theoret. Comput. Sci. 23 (1983), 69-82.

4. A. Carpi. On the repetition threshold for large alphabets. In R. Královič and P. Urzyczyn, eds., Proc. MFCS 2006, Lect. Notes in Comput. Sci. \#3162, SpringerVerlag, 2006, pp. 226-237.

5. F. Dejean, Sur un théorème de Thue, J. Comb. Theory. Ser. A 13 (1972), 90-99.

6. R. C. Entringer, D. E. Jackson, and J. A. Schatz. On nonrepetitive sequences. J. Combin. Theory. Ser. A 16 (1974), 159-164.

7. G. M. Landau and J. P. Schmidt. An algorithm for approximate tandem repeats. In A. Apostolico, M. Crochemore, Z. Galil, and U. Manber, eds., Combinatorial Pattern Matching, 4th Annual Symposium, CPM 93. Lect. Notes in Comput. Sci. \# 684, Springer-Verlag, 1993, pp. 120-133.

8. R. Kolpakov and G. Kucherov. Finding approximate repetitions under Hamming distance. Theor. Comput. Sci. 303 (2003), 135-156.

9. M. Mohammad-Noori and J. D. Currie. Dejean's conjecture and Sturmian words. European J. Combin. 28 (2007), 876-890.

10. J. Moulin-Ollagnier. Proof of Dejean's conjecture for alphabets with 5, 6, 7, 8, 9, 10 and 11 letters. Theoret. Comput. Sci. 95 (1992), 187-205.

11. T. Nagell (ed.). Selected Mathematical Papers of Axel Thue. Universitetsforlaget, Oslo, 1977.

12. J.-J. Pansiot. A propos d'une conjecture de F. Dejean sur les répétitions dans les mots. Discrete Appl. Math. 7 (1984), 297-311.

13. A. Thue. Über unendliche Zeichenreihen. Norske vid. Selsk. Skr. Mat. Nat. Kl. 7 (1906), 1-22. Reprinted in [11, pp. 139-158].

14. A. Thue. Über die gegenseitige Lage gleicher Teile gewisser Zeichenreihen. Norske vid. Selsk. Skr. Mat. Nat. Kl. 1 (1912), 1-67. Reprinted in [11, pp. 413-478]. 